\title{
Solving fuzzy Volterra integro-differential equation by fuzzy differential transform method
}

\author{
N. Mikaeilvand ${ }^{1}$ S. Khakrangin ${ }^{2}$ T. Allahviranloo ${ }^{3}$ \\ ${ }^{1}$ Department of Mathematics, Ardabil Branch, Islamic Azad University, Ardabil, Iran. \\ ${ }^{2,3}$ Department of Mathematics, Science and Research Branch, Islamic Azad University, Tehran, Iran.
}

\begin{abstract}
In this study, differential transform method (DTM) is applied to fuzzy integro-differential equation. The concept of generalized $\mathrm{H}$ differentiability is used. If the equation has a solution in terms of the series expansion of known functions; this powerful method catches the exact solution. Some numerical examples are also given to illustrate the superiority of the method. All rights reserved.
\end{abstract}

Keywords: Fuzzy differential transform method ; Integro-differential equation; Taylor series.

\section{Introduction}

When a physical system is modeled under the differential sense; It finally gives a fuzzy differential equation, a fuzzy integral equation or a fuzzy integro-differential equation and hence, the solution of integro-differential equations have a major role in the fields of science and engineering. Nonlinear integro-differential equations are usually hard to solve analytically and exact solutions are scarce. Therefore, they have been of great interest by several authors. The technique that we used is the fuzzy differential transform method ( FDTM ), which is based on Taylor series expansion. Differential transform method is different from the traditional high order Taylor series method. When requires symbolic computation of necessary derivatives of the data function and is computationally expensive for higher order. Intrinsically, the differential transformation method evaluates the approximate solution by the finite Taylor series. But, in the differential transform method the derivative is not computed directly. Instead, the relative derivatives are calculated by an iteration procedure. It is introduced by Zhou [5] in a study about electrical circuits. In this way, Allahviranloo et. al [1] proposed FDTM for solving first order fuzzy differential equation under strongly H-differentiability. Moreover, Arikoglu et. al [2] has been proposed differential transform method for solving integrodifferential equations. It gives exact values of the nth derivative of an analytical function at a point in a fast manner. DTM is a semi analyticalnumerical technique that depends on Taylor series.
In this study, DTM is applied to fuzzy integrodifferential equations.

The structure of paper is organized as follows:

In section 2, some basic definitions and results which will be used later are brought. In section 3 , we shall propose fuzzy differential transform method for solving fuzzy Volterra integrodifferential equation with separable kernels. Then the proposed method is implemented to two numerical-analytical examples in section 4 and finally, conclusion is drawn in section 5 .

\section{Preliminaries}

An arbitrary fuzzy number $\widetilde{u}$ is represented by an ordered pair of functions $(\underline{u}(r), \bar{u}(r)) ; \quad 0 \leq r \leq 1$ which satisfy the following requirements is represented.

(i) $\underline{u}(r)$ is a bounded monotonic increasing left continuous function;

(ii) $\bar{u}(r)$ is a bounded monotonic decreasing left continuous function;

(iii) $\underline{u}(r) \leq \bar{u}(r), 0 \leq r \leq 1$.

A crisp number $k$ is simply represented by $\bar{u}(r)=$ $\underline{u}(r)=k ; \quad 0 \leq r \leq 1$, and called singleton. Let $E$ be the set of all upper semicontinuous normal convex fuzzy numbers with bounded r-level intervals. It means that if $v \in E$ then the r-level set

$$
[v]^{r}=\{s \mid v(s) \geq r, 0<r \leq 1\}
$$

is a closed bounded interval which is denoted by

$$
[v]^{r}=[\underline{v}(r), \bar{v}(r)] .
$$

For arbitrary $\widetilde{u}=(\underline{u}(r), \bar{u}(r)), \widetilde{v}=(\underline{v}(r), \bar{v}(r))$ and scalar $k$ we define addition $(\widetilde{u}+\widetilde{v})$, subtraction and scalar multiplication by $k$ as

Addition:

$$
(\underline{u+v})(r)=\underline{u}(r)+\underline{v}(r), \quad(\overline{u+v})(r)=\bar{u}(r)+\bar{v}(r),
$$

subtraction:

$$
(\underline{u-v})(r)=\underline{u}(r)-\bar{v}(r), \quad(\overline{u-v})(r)=\bar{u}(r)-\underline{v}(r),
$$

scalar multiplication:

$$
\widetilde{k u}= \begin{cases}(k \underline{u}(r), k \bar{u}(r)), & k \geq 0, \\ (k \bar{u}(r), k \underline{u}(r)), & k<0 .\end{cases}
$$


For two arbitrary fuzzy numbers $\widetilde{x}=(\underline{x}(r), \bar{x}(r))$ and $\widetilde{y}=(y(r), \bar{y}(r)), \widetilde{x}=\widetilde{y}$ if and only if $\underline{x}(r)=y(r)$ and $\bar{x}(r)=\bar{y}(r)$. Since each $y \in R$ can be regarded as a fuzzy number $\widetilde{y}$ defined by

$$
\widetilde{y}(t)=\left\{\begin{array}{cc}
1, & \text { ift }=y, \\
0, & \text { ift } \neq y .
\end{array}\right.
$$

The Hausdorff distance between fuzzy numbers given by $D: E \times E \rightarrow R_{+} \bigcup\{0\}$.

$$
D(u, v)=\sup _{r \in[0,1]} \operatorname{Max}\{|\underline{u}(r)-\underline{v}(r)|,|\bar{u}(r)-\bar{v}(r)|\}
$$

It is easy to see that $D$ is a metric in $E$ and has the following properties (see [7]).

(i) $D(u \oplus w, v \oplus w)=D(u, v), \forall u, v, w \in E$.

(ii) $D(k \odot u, k \odot v)=|k| D(u, v), \forall k \in R, u, v \in E$.

(iii) $D(u \oplus v, u \oplus e) \leq D(u, w)+D(v, e), \forall u, v, w, e \in$ $E$,

(iv) $(D, E)$ is a complete metric space.

Definition 1 [4] Let $f: R \longrightarrow E$ be a fuzzy valued function. If for arbitrary fixed $t_{0} \in R$ and $\epsilon>0$. a $\delta>0$ such that $\left|t-t_{0}\right|<\delta \Longrightarrow D\left(f(t), f\left(t_{0}\right)\right)<\epsilon$ $f$ is said to be continuous.

It is well-known that the H-derivative (differentiability in the sense of Hukuhara) for fuzzy mappings was initially introduced by Puri and Ralescu [6]. It is based on the H-difference of sets:

Definition 2 Let $x, y \in E$. If there exists $z \in E$ such that $x=y+z$, then $z$ is called the H-difference of $x$ and $y$ and it is denoted by $x \ominus y$.

In this paper, the following definition which was introduced by Chalco-Cano et. al [3] is considered.

Definition 3 Let $f:(a, b) \rightarrow E$ and $x_{0} \in(a, b)$. We say that $f$ is differentiable at $x_{0}$. If there exists an element $f^{\prime}\left(x_{0}\right) \in E$, such that

(1) for all $h>0$ sufficiently near to $0, \exists f\left(x_{0}+h\right) \ominus$ $f\left(x_{0}\right), \exists f\left(x_{0}\right) \ominus f\left(x_{0}-h\right)$ and the limits (in the metric $D)$

$$
\begin{aligned}
& \lim _{h \rightarrow 0^{+}} \frac{f\left(x_{0}+h\right) \ominus f\left(x_{0}\right)}{h} \\
& \quad=\lim _{h \rightarrow 0^{+}} \frac{f\left(x_{0}\right) \ominus f\left(x_{0}-h\right)}{h}=f^{\prime}\left(x_{0}\right)
\end{aligned}
$$

or

(2) for all $h<0$ sufficiently near to $0, \exists f\left(x_{0}+h\right) \ominus$ $f\left(x_{0}\right), \exists f\left(x_{0}\right) \ominus f\left(x_{0}-h\right)$ and the limits (in the metric D)

$$
\begin{aligned}
& \lim _{h \rightarrow 0^{-}} \frac{f\left(x_{0}+h\right) \ominus f\left(x_{0}\right)}{h} \\
& \quad=\lim _{h \rightarrow 0^{-}} \frac{f\left(x_{0}\right) \ominus f\left(x_{0}-h\right)}{h}=f^{\prime}\left(x_{0}\right) .
\end{aligned}
$$

In the special case, when $f$ is a fuzzy-valued function, we have the following result:

Theorem 1 [3] Let $f: R \rightarrow E$ be a function and denote $f(t)=(\underline{f}(t, r), \bar{f}(t, r))$. For each $r \in[0,1]$,
(1) if $f$ is differentiable in the first form (1) in Definition 3 , then $f(t, r)$ and $\bar{f}(t, r)$ are differentiable functions and $f^{\prime}(t)=\left(f^{\prime}(t, r), \bar{f}^{\prime}(t, r)\right)$

(2) if $f$ is differentiable in the second form (2) in Definition 3, then $f(t, r)$ and $\bar{f}(t, r)$ are differentiable functions and $f^{\prime}(t)=$ $\left(\bar{f}^{\prime}(t, r), f^{\prime}(t, r)\right)$.

Moreover, we can consider the second order of later type of H-differentiability as following:

Theorem 2 Let $f: R \rightarrow E$ be a function and denote $f(t)=(\underline{f}(t, r), \bar{f}(t, r))$. For each $r \in[0,1]$, then

(A1) if $f$ and $f^{\prime}$ are differentiable in the first form (1) or if $f$ and $f^{\prime}$ are differentiable in the second form (2) in Definition 3, then $f(t, r)$ and $\bar{f}(t, r)$ are differentiable functions and $f^{\prime \prime}(t)=$ $\left(f^{\prime \prime}(t, r), \bar{f}^{\prime \prime}(t, r)\right)$

(A2) if $f$ is differentiable in the first form (1) and $f^{\prime}$ is differentiable in the second form (2)or if $f$ is differentiable in the second form (2) and $f^{\prime}$ is differentiable in the first form (1) in Definition 3 , then $f(t, r)$ and $\bar{f}(t, r)$ are differentiable functions and $f^{\prime \prime}(t)=\left(\bar{f}^{\prime \prime}(t, r), \underline{f}^{\prime \prime}(t, r)\right)$.

Proof:It is straightforward.

Definition 4 The transformation of the nth derivative of a function in one variable is as follows:

$$
F(k)=\frac{1}{k !}\left[\frac{d^{k}}{d x^{k}} f(x)\right]_{x=x_{0}}
$$

and the inverse transformation is defined as

$$
f(x)=\sum_{k=0}^{\infty} F(k)\left(x-x_{0}\right)^{k}
$$

\section{Fuzzy Volterra Integro-Differential Equation With Separable Kernels}

In this section, we will investigate solution of fuzzy Volterra integro-differential equations with separable kernels. Let $u(x)$ be a fuzzy-valued function to be solved for, $f(x)$ is given known function and $k(x, t)$ is a known real-valued integral kernel. The fuzzy Volterra integro-differential equation explain in the form

$$
u^{\prime}(x)=f(x)+\int_{0}^{x} k(x, t) u(x) d t
$$

where $f(x): R \rightarrow E$ and $x \in[a, b], b<\infty$.

In addition, FDTM is used for solving Eq.(1) with separable kernels i.e

$$
k(x, t)=\sum_{j=0}^{n} Q_{j}(x) P_{j}(t)
$$

Let us consider $u^{\prime}(x)=F(x, t)$ where 


$$
\begin{aligned}
& \underline{u}(x)=\underline{u}\left(x_{0}\right)+\int_{x_{0}}^{x} \underline{F}(x, t) d x, \\
& \bar{u}(x)=\bar{u}\left(x_{0}\right)+\int_{x_{0}}^{x} \bar{F}(x, t) d x
\end{aligned}
$$

Definition 5 Let us consider $x(t)$ is differentiable of order $k$ over time domain $T$, then

$$
\begin{aligned}
& \left.\underline{X}_{i}(k, r)=\frac{d^{k} \underline{x}(t, r)}{d t^{k}}\right]_{t=t_{i}}, \quad \forall k \in K=0,1, \ldots \\
& \left.\bar{X}_{i}(k, r)=\frac{d^{k} \bar{X}(t, r)}{d t^{k}}\right]_{t=t_{i}},
\end{aligned}
$$

when $x(t)$ is (1)-differentiable and

when $k$ is odd

$$
\left.\left.\underline{X}_{i}(k, r)=\frac{d^{k} \bar{x}(t, r)}{d t^{k}}\right]_{t=t_{i}}, \quad \bar{X}_{i}(k, r)=\frac{d^{k} \underline{x}(t, r)}{d t^{k}}\right]_{t=t_{i}}
$$

when $k$ is even

$$
\left.\left.\underline{X}_{i}(k, r)=\frac{d^{k} x(t, r)}{d t^{k}}\right]_{t=t_{i}}, \quad \bar{X}_{i}(k, r)=\frac{d^{k} \bar{x}(t, r)}{d t^{k}}\right]_{t=t_{i}}
$$

when $x(t)$ is (2)-differentiable.

Notice that $\underline{X}_{i}(t, r)$ and $\bar{X}_{i}(t, r)$ are called the lower and upper spectrum of $x(t)$ at $t=t_{i}$, respectively. So, if $x(t)$ be (1)-differentiable then $x(t)$ can be represented as follows:

$$
\begin{aligned}
& \underline{x}(t, r)=\sum_{k=0}^{\infty} \frac{\left(t-t_{i}\right)^{k}}{k !} \underline{X}(k, r), k \in K, 0 \leq r \leq 1 \\
& \bar{x}(t, r)=\sum_{k=0}^{\infty} \frac{\left(t-t_{i}\right)^{k}}{k !} \bar{X}(k, r), k \in K, 0 \leq r \leq 1
\end{aligned}
$$

and if $x(t)$ be (2)-differentiable then $x(t)$ can be represented as follows:

$$
\begin{aligned}
\underline{x}(t, r) & =\sum_{k=0}^{\infty} \frac{\left(t-t_{i}\right)^{k}}{k !} \bar{X}(k, r) \\
& +\sum_{k=0}^{\infty} \frac{\left(t-t_{i}\right)^{k}}{k !} \underline{X}(k, r), \quad 0 \leq r \leq 1 \\
\bar{x}(t, r) & =\sum_{k=0}^{\infty} \frac{\left(t-t_{i}\right)^{k}}{k !} \underline{X}(k, r) \\
& +\sum_{k=0}^{\infty} \frac{\left(t-t_{i}\right)^{k}}{k !} \bar{X}(k, r), 0 \leq r \leq 1
\end{aligned}
$$

The mentioned equations are known as the inverse transformation of $X(k, r)$. If $X(k, r)$ is defined as

$$
\begin{aligned}
& \underline{X}(k, r)=M(k)\left[\frac{d^{k}\left(\frac{x(t, r))}{d t^{k}}\right]_{t=t_{0}},}{\bar{X}(k, r)=M(k)\left[\frac{d^{k}(\overline{x(t, r)})}{d t^{k}}\right]_{t=t_{0}}}\right.
\end{aligned}
$$

when is $x(t)$ is (1)-differentiable and

$$
\begin{aligned}
& \text { for } k \text { is odd } \\
& \underline{X}(k, r)=M(k)\left[\frac{d^{k}(\overline{x(t, r)})}{d t^{k}}\right]_{t=t_{0}}, \\
& \bar{X}(k, r)=M(k)\left[\frac{d^{k}(\underline{x(t, r))}}{d t^{k}}\right]_{t=t_{0}} \\
& \text { for } k \text { is even } \\
& \underline{X}(k, r)=M(k)\left[\frac{d^{k}(x(t, r))}{d t^{k}}\right]_{t=t_{0}}, \\
& \bar{X}(k, r)=M(k)\left[\frac{d^{k}(\overline{x(t, r)})}{d t^{k}}\right]_{t=t_{0}}
\end{aligned}
$$

when $x(t)$ is (2)-differentiable then, the function $x(t)$ can be represented as follows:

$$
\begin{array}{ll}
\underline{x}(t, r)=\sum_{k=0}^{\infty} \frac{\left(t-t_{0}\right)^{k}}{k !} \frac{X}{\bar{M}(t, r)} & \\
\bar{x}(t, r) & =\sum_{k=0}^{\infty} \frac{\left(t-t_{0}\right)^{k}}{k !} \frac{\bar{X}(t, r)}{M(k)},
\end{array}
$$

when $x(t)$ is (1)-differentiable and if $x(t)$ be (2)differentiable we get the following:

$$
\begin{aligned}
\underline{x}(t, r) & =\left(\sum_{k=0, \text { odd }}^{\infty} \frac{\left(t-t_{0}\right)^{k}}{k !} \frac{\bar{X}(t, r)}{M(k)}\right. \\
& \left.+\sum_{k=0, \text { even }}^{\infty} \frac{\left(t-t_{0}\right)^{k}}{k !} \frac{X(t, r)}{\bar{M}(k)}\right), \\
\bar{x}(t, r) & =\left(\sum_{k=0, \text { odd }}^{\infty} \frac{\left(t-t_{0}\right)^{k}}{k !} \frac{X(t, r)}{\bar{M}(k)}\right. \\
& \left.+\sum_{k=0, \text { even }}^{\infty} \frac{\left(t-t_{0}\right)^{k}}{k !} \frac{\bar{X}(t, r)}{M(k)}\right),
\end{aligned}
$$

where $M(k)>0, M(k)$ is called the weighting factor and in this paper, is applied $M(k)=\frac{H^{k}}{k !}$, where $\mathrm{H}$ is the time horizon on interest. So, if $x(t)$ be (1)-differentiable. Then,

$$
\begin{gathered}
\underline{X}(k, r)=\frac{H^{k}}{k !} \frac{d^{k} \underline{x}(t, r)}{d t^{k}}, \\
\bar{X}(k, r)=\frac{H^{k}}{k !} \frac{d^{k} \bar{x}(t, r)}{d t^{k}}, \\
k \in K, 0 \leq r \leq 1
\end{gathered}
$$

and if $x(t)$ be (2)-differentiable,

$$
k \text { is odd, }
$$

$$
\begin{aligned}
& \underline{X}(k, r)=\frac{H^{k}}{k !} \frac{d^{k} \bar{x}(t, r)}{d t^{k}}, \\
& \bar{X}(k, r)=\frac{H^{k}}{k !} \frac{d^{k} \underline{x}(t, r)}{d t^{k}},
\end{aligned}
$$

$k$ is even,

$$
\begin{aligned}
& \underline{X}(k, r)=\frac{H^{k}}{k !} \frac{d^{k} \underline{\underline{x}}(t, r)}{d t^{k}}, \\
& \bar{X}(k, r)=\frac{H^{k}}{k !} \frac{d^{k} \bar{x}(t, r)}{d t^{k}},
\end{aligned}
$$


Using the fuzzy differential transform, a fuzzy differential equation in the domain of interest can be transformed to an algebraic equation in the domain $k$ and $x(t)$ can be obtained as the finite-term Taylor series plus a reminder as following $k \in K, 0 \leq r \leq 1$ :

$$
\begin{aligned}
\underline{x}(t, r) & =\sum_{k=0}^{N} \frac{\left(t-t_{0}\right)^{k}}{k !} \frac{\underline{X}(t, r)}{M(k)}+R_{N+1}(t) \\
& =\sum_{k=0}^{N}\left(\frac{t-t_{0}}{H}\right)^{k} \underline{X}(t, r)+R_{N+1}(t), \\
\bar{x}(t, r) & =\sum_{k=0}^{N} \frac{\left(t-t_{0}\right)^{k}}{k !} \frac{\bar{X}(t, r)}{M(k)}+R_{N+1}(t) \\
& =\sum_{k=0}^{N}\left(\frac{t-t_{0}}{H}\right)^{k} \bar{X}(t, r)+R_{N+1}(t),
\end{aligned}
$$

when is $x(t)$ is (1)-differentiable and $k \in K, 0 \leq r \leq$ 1

$$
\begin{aligned}
& \underline{x}(t, r)=\left(\sum_{k=1, o d d}^{N} \frac{\left(t-t_{i}\right)^{k}}{k !} \frac{\bar{X}(t, r)}{M(k)}\right. \\
& \left.+\sum_{k=0, \text { even }}^{N} \frac{\left(t-t_{i}\right)^{k}}{k !} \frac{\underline{X}(t, r)}{M(k)}\right)+R_{N+1}(t) \\
& =\sum_{k=1, o d d}^{N}\left(\frac{t-t_{i}}{H}\right)^{k} \bar{X}(t, r) \\
& +\sum_{k=0, \text { even }}^{N}\left(\frac{t-t_{i}}{H}\right)^{k} \underline{X}(t, r)+R_{N+1}(t), \\
& \bar{x}(t, r)=\left(\sum_{k=1, o d d}^{N} \frac{\left(t-t_{i}\right)^{k}}{k !} \frac{X(t, r)}{M(k)}\right. \\
& \left.+\sum_{k=0, \text { even }}^{N} \frac{\left(t-t_{i}\right)^{k}}{k !} \frac{\bar{X}(t, r)}{M(k)}\right)+R_{N+1}(t) \\
& =\sum_{k=1, o d d}^{N}\left(\frac{t-t_{i}}{H}\right)^{k} \underline{X}(t, r) \\
& +\sum_{k=0, \text { even }}^{N}\left(\frac{t-t_{i}}{H}\right)^{k} \bar{X}(t, r)+R_{N+1}(t)
\end{aligned}
$$

when is $x(t)$ is (2)-differentiable.

The objective of this section is to find the solution of Eq. (1) at the equally spaced grid points $\left[t_{0}, \ldots, t_{N}\right]$ where $t_{i}=a+i h$ for each $i=0,1, \ldots, N$ and $h=\frac{b-a}{N}$. That is, the domain of interest is divided to $N$ sub-domain and the fuzzy approximation functions in each sub-domain are $x_{i}(t, r)$ for $i=0,1, \ldots, N-1$, respectively. From the initial conditions the following can obtained:

$\underline{X}(0, r)=\underline{x}(0, r), \quad \bar{X}(0, r)=\bar{x}(0, r), \quad 0 \leq r \leq 1$
In the first sub-domain, $\underline{x}(t, r)$ and $\bar{x}(t, r)$ can be described by $\underline{x}(0, r)=\underline{x}_{0}(r)$ and $\bar{x}(0, r)=\bar{x}_{o}(r)$ respectively. They can be represented in terms of their n-th order Taylor series with respect to do. That is

$$
\begin{aligned}
\underline{x}\left(t_{0}, r\right) & =\underline{X}_{0}(0, r)+\underline{X}_{0}(1, r)\left(t-t_{0}\right) \\
& +\underline{X}_{0}(2, r)\left(t-t_{0}\right)^{2}+\ldots+\underline{X}_{0}(N, r)\left(t-t_{0}\right)^{N} \\
\bar{x}\left(t_{0}, r\right) & =\bar{X}_{0}(0, r)+\bar{X}_{0}(1, r)\left(t-t_{0}\right) \\
& +\bar{X}_{0}(2, r)\left(t-t_{0}\right)^{2}+\ldots+\bar{X}_{0}(N, r)\left(t-t_{0}\right)^{N}
\end{aligned}
$$

Additionally, using Taylor series for $x\left(t_{1}, r\right)$ leads to obtain:

$$
\begin{aligned}
\underline{x}\left(t_{1}, r\right) & =\underline{X}_{0}(0, r)+\underline{X}_{0}(1, r)\left(t_{1}-t_{0}\right)+\underline{X}_{0}(2, r)\left(t_{1}-t_{0}\right)^{2} \\
& +\cdots+\underline{X}_{0}(N, r)\left(t_{1}-t_{0}\right)^{N} \\
& =\sum_{j=0}^{N} \underline{X}_{0}(j, r) h^{j} \\
\bar{x}\left(t_{1}, r\right) & =\bar{X}_{0}(0, r)+\bar{X}_{0}(1, r)\left(t_{1}-t_{0}\right)+\bar{X}_{0}(2, r)\left(t_{1}-t_{0}\right)^{2} \\
& +\cdots+\bar{X}_{0}(N, r)\left(t_{1}-t_{0}\right)^{N} \\
& =\sum_{j=0}^{N} \bar{X}_{0}(j, r) h^{j} .
\end{aligned}
$$

The final value $x_{0}\left(t_{1}\right)$ of the first sub-domain is the initial value of the second sub-domain ,i.e. $x_{1}\left(t_{1}, r\right)=X_{1}(0)=x_{0}\left(t_{1}, r\right)$. In a similar manner $x\left(t_{2}, r\right)$ can be represented as

$$
\begin{aligned}
\underline{x}\left(t_{2}, r\right) \approx \underline{x}_{1}\left(t_{2}, r\right) & =\underline{X}_{1}(0, r) \\
& +\underline{X}_{1}(1, r)\left(t_{2}-t_{1}\right)+\underline{X}_{1}(2, r)\left(t_{2}-t_{1}\right)^{2} \\
& +\ldots+\underline{X}_{1}(N, r)\left(t_{2}-t_{1}\right)^{N} \\
& =\sum_{j=0}^{N} \underline{X}_{1}(j, r) h^{j}, \\
\bar{x}\left(t_{1}, r\right) \approx \bar{x}_{1}\left(t_{2}, r\right) & =\bar{X}_{1}(0, r) \\
& +\bar{X}_{1}(1, r)\left(t_{2}-t_{1}\right)+\bar{X}_{1}(2, r)\left(t_{2}-t_{1}\right)^{2} \\
& +\ldots+\bar{X}_{1}(N, r)\left(t_{2}-t_{1}\right)^{N} \\
& =\sum_{j=0}^{N} \bar{X}_{1}(j, r) h^{j} .
\end{aligned}
$$

Hence, the solution on the gird points $t_{i+1}$ can be 
obtained as follows:

$$
\begin{aligned}
\underline{x}\left(t_{i+1}, r\right) \approx \underline{x}_{i}\left(t_{i+1}, r\right) & =\underline{X}_{i}(0, r)+\underline{X}_{i}(1, r)\left(t_{i+1}-t_{i}\right) \\
& +\underline{X}_{i}(2, r)\left(t_{i+1}-t_{i}\right)^{2} \\
& +\ldots+\underline{X}_{i}(N, r)\left(t_{i+1}-t_{i}\right)^{N} \\
& =\sum_{j=0}^{N} \underline{X}_{i}(j, r) h^{j}, \\
\bar{x}\left(t_{i+1}, r\right) \approx \bar{x}_{i}\left(t_{i+1}, r\right) & =\bar{X}_{i}(0, r)+\bar{X}_{i}(1, r)\left(t_{i+1}-t_{i}\right) \\
& +\bar{X}_{i}(2, r)\left(t_{i+1}-t_{i}\right)^{2} \\
& +\ldots+\bar{X}_{i}(N, r)\left(t_{i+1}-t_{i}\right)^{N} \\
& =\sum_{j=0}^{N} \bar{X}_{i}(j, r) h^{j} .
\end{aligned}
$$

Now, we investigate the important properties of fuzzy transform of fuzzy-valued functions.

Theorem 3 Let us consider $u(t)$ and $v(t)$ are fuzzy-valued functions, then

1. If $f(t)=u(t) \pm v(t)$ then $F(k)=U(k) \pm$ $V(k), k \in K$

2. If $f(t)=u(t) \ominus v(t)$ then $F(k)=U(k) \ominus$ $V(k), k \in K$

3. If $f(t)=\left(d^{m} z(t) / d t^{m}\right)$ then

$$
F(k)=\frac{(m+k) !}{k !} Z(k+m)
$$

4. If $f(t)=\alpha w(t)$ then $F(k)=\alpha W(k)$

5. If $f(t)=t^{m}$ then $F(k)=\delta(k-m)$

6. If $f(t)=\exp (\lambda t)$ then $F(k)=\lambda^{k} / k$ !

7. If $f(t)=(1+t)^{m}$ then

$$
F(k)=\frac{m(m-1)-(m-k-1)}{k !}
$$

8. If $f(t)=\sin (w t+\alpha)$ then

$$
F(k)=\frac{w^{k}}{k !} \sin \left(\pi \frac{k}{2 !}+\alpha\right)
$$

9. If $f(t)=\cos (w t+\alpha)$ then

$$
F(k)=\frac{w^{k}}{k !} \cos \left(\pi \frac{k}{2 !}+\alpha\right)
$$

Proof:Using definition 5 the proofs are obvious.

Theorem 4 Suppose that $U(t)$ and $G(t)$ are differential transformations of the functions $u(t)$ and $g(t)$ (is real-values function), respectively, then we have the following:

1. If $f(x)=\int_{x_{0}}^{x} u(t) d t$ then, $F(k, r)=\frac{U(k-1, r)}{k}$, where $k \geq 1$.

2. If $f(x)=\int_{x_{0}}^{x} g(t) u(t) d t$ then

$$
\begin{gathered}
F(k ; r)=\sum_{l=0}^{k-1} G(l) \frac{U(k-l-1 ; r)}{k}, \\
F(0)=0,0 \leq r \leq 1,
\end{gathered}
$$

3. If $f(x)=g(x) \int_{x_{0}}^{x} u(t) d t$ then

$$
\begin{gathered}
F(k ; r)=\sum_{l=0}^{k-1} G(l) \frac{U(k-l-1 ; r)}{k-1}, \\
F(0)=0,0 \leq r \leq 1 \\
f(x)=g(x) u(x) \text { then } \\
F(k ; r)=\sum_{l=0}^{k} G(l) U(k-l ; r), 0 \leq r \leq 1
\end{gathered}
$$$$
\text { 4. If } f(x)=g(x) u(x) \text { then }
$$

Proof:The proofs using definition 5 are obvious.

\section{Numerical Examples}

In this section, we will investigate the solution of fuzzy Volterra integro-differential equations using fuzzy differential transform method to show the utility of proposed method.

Example 1 Let us consider the following fuzzy Volterra integro-differential equation:

$$
\begin{gathered}
u(x)=(1+x)(r+1, r-2)+\int_{0}^{x} u(t) d t, \\
u(0)=(0,0), \quad u^{\prime}(0)=(r+1, r-2),
\end{gathered}
$$

based on Theorem 4 and using properties of FDTM . We have the following for all $0 \leq k \leq$ $1, k \geq 1$ :

$\underline{U}(k+1 ; r)=\left[(\delta(k)+\delta(k-1))(r+1)+\frac{\underline{U}(k-1, r)}{k}\right] \frac{k}{(k+1) !}$

and

$\bar{U}(k+1 ; r)=\left[(\delta(k)+\delta(k-1))(r-2)+\frac{\bar{U}(k-1, r)}{k}\right] \frac{k}{(k+1) !}$

where $\underline{U}(0 ; r)=0, \quad \underline{U}(1, r)=r+1, \quad \bar{U}(0, r)=0$ and $\bar{U}(1, r)=r-2$. Consequently, we obtain:

$$
\begin{array}{llll}
\underline{U}(2 ; r)=\frac{r+1}{2 !}, & \underline{U}(3 ; r)=\frac{r+1}{3 !}, & & \underline{U}(4 ; r)=\frac{r+1}{4 !}, \\
\underline{U}(5 ; r)=\frac{r+1}{5 !}, & \underline{U}(6 ; r)=\frac{r+1}{6 !}, & \underline{U}(7 ; r)=\frac{r+1}{7 !}, \\
\underline{U}(8 ; r)=\frac{r+1}{8 !}, & \underline{U}(9 ; r)=\frac{r+1}{9 !}, & \underline{U}(10 ; r)=\frac{r+1}{10 !},
\end{array}
$$

and

$$
\begin{array}{lll}
\bar{U}(2 ; r)=\frac{r-2}{2 !}, & \bar{U}(3 ; r)=\frac{r-2}{3 !}, & \bar{U}(4 ; r)=\frac{r-2}{4 !}, \\
\bar{U}(5 ; r)=\frac{r-2}{5 !}, & \bar{U}(6 ; r)=\frac{r-2}{6 !}, & \bar{U}(7 ; r)=\frac{r-2}{7 !}, \\
\bar{U}(8 ; r)=\frac{r-2}{8 !}, & \bar{U}(9 ; r)=\frac{r-2}{9 !}, & \bar{U}(10 ; r)=\frac{r-2}{10 !},
\end{array}
$$

therefore, the solution of fuzzy Volterra integrodifferential equation (10) will be as following

$$
\begin{aligned}
u(x) & =(r+1, r-2)\left(x+x^{2} / 2 !+x^{3} / 3 !+x^{4} / 4 !\right. \\
& \left.+x^{5} / 5 !+x^{6} / 6 !+x^{7} / 7 !+x^{8} / 8 !+\ldots\right) \\
& =(r+1, r-2)\left(e^{x}-1\right)
\end{aligned}
$$

which is the exact solution of fuzzy Volterra integrodifferential equation (10). 
Example 2 We consider the following fuzzy integro-differential equation:

$$
\begin{gathered}
u^{\prime}(x)=(r-1,1-r)+\int_{0}^{x} u(t) d t, \\
u(0)=(0,0), \quad u^{\prime}(0)=(r-1,1-r)
\end{gathered}
$$

By applying Theorem (4) and using properties of FDTM, the following relation is obtained for $0 \leq$ $r \leq 1$ :

$\underline{U}(k+1, r)=\left[(r-1) \delta(k)+\frac{U(k-1, r)}{k}\right] \frac{k !}{(k+1) !}$

and

$\bar{U}(k+1, r)=\left[(1-r) \delta(k)+\frac{\bar{U}(k-1, r)}{k}\right] \frac{k !}{(k+1) !}$

By using Theorem 3.1 and problem condition, we have

$$
\begin{aligned}
& \underline{U}(0, r)=0, \underline{U}(1, r)=(r-1), \\
& \bar{U}(0, r)=0, \quad \bar{U}(1, r)=(1-r) .
\end{aligned}
$$

Utilizing the above relation, we obtain:

$$
\begin{array}{ccc}
\underline{U}(2, r)=0, & \underline{U}(3, r)=\frac{r-1}{3 !}, & \underline{U}(4, r)=0, \\
\underline{U}(5, r)=\frac{r-1}{5 !}, & \underline{U}(6, r)=0, & \underline{U}(7, r)=\frac{r-1}{7 !}, \\
\underline{U}(8, r)=0, & \underline{U}(9, r)=\frac{r-1}{9 !}, & \underline{U}(10, r)=0,
\end{array}
$$

and

$$
\begin{array}{ccc}
\bar{U}(2, r)=0, & \bar{U}(3, r)=\frac{1-r}{3 !}, & \bar{U}(4, r)=0, \\
\bar{U}(5, r)=\frac{1-r}{5 !}, & \bar{U}(6, r)=0, & \bar{U}(7, r)=\frac{1-r}{7 !}, \\
\bar{U}(8, r)=0, & \bar{U}(9, r)=\frac{1-r}{9 !}, & \bar{U}(10, r)=0,
\end{array}
$$

Then, the following series solution is evaluated:

$$
\begin{aligned}
u(x) & =(r-1,1-r)\left(x+\frac{x^{3}}{3 !}+\frac{x^{5}}{5 !}+\frac{x^{7}}{7 !}+\ldots\right) \\
& =(r-1,1-r) \sinh x
\end{aligned}
$$

Which is the exact solution of the fuzzy integrodifferential equation

\section{Conclusion}

In this paper, FDTM is proposed for solving fuzzy Volterra integro-differential equation with separable kernels. Differential transform method is different from the traditional high order Taylor series method, which requires symbolic computation of necessary derivatives of the data function and is computationally expensive for higher order. We introduced new theorems for FDTM to solve the fuzzy integro-differential equations. We first gave their proofs and then applied to fuzzy integrodifferential equations. Also, we used the concept of H-derivatives for fuzzy mappings. Some examples were examined using FDTM and the results have shown remarkable performance.

\section{References}

[1] T. Allahviranloo, N. A. Kiani, Solving fuzzy differential equations by differential transformation method, Information Science 179:956-966, Elsevier, 2009.

[2] A. Arikoglu, I. Ozkol, Solution of boundary value problems for integro-differential equations by using differential transform method, Applied Mathematics and Computation 168:1145-1158, Elsevier, 2005.

[3] Y.Chalco-Cano, H. Roman- Flores, On new solutions of fuzzy differential equations. Chaos. Solitons and Fractals 38:112-119, Elsevier, 2006.

[4] M. Friedman, M. Ma, A. Kandel, Numerical solution of fuzzy differential and integral equations, Fuzzy Sets and Systems 106:35-48, Elsevier, 1999.

[5] J. K. Zhou, Differential transformation and Its Application for Electrical Circuits, Huazhong University Press, Wuhan, China, 1986.

[6] M. L. Puri. D. Ralescu, Differential for fuzzy function. J. Math. Appl. 91:552-558, 1983.

[7] M. L. Puri. D. Ralescu, Fuzzy random variables, J. Math. Appl. 114:409-422, 1986. 